\title{
Knowledge Management in Public Web Call Centres
}

\author{
Vincenzo Ambriola, Silvia Bertagnini, and Letizia Pratesi \\ Università di Pisa - Dipartimento di Informatica, Via F. Buonarroti, 2, \\ 56127 - PISA (Italy), phone +39 $0502212\{751,784,784\}$ \\ \{ambriola, bertagni, pratesi\}@di.unipi.it
}

\begin{abstract}
The increased use of Internet has accelerated the evolution of call centres: from simple centres with few telephone lines to sophisticated web call centres that give users different instruments to search information. In this vision the success of a web call centre depends more and more on knowledge. Without enough knowledge, call centre staff cannot provide the level of service that users are demanding. Recently, the idea of using web call centres is gaining popularity in the public sector too. This paper presents and discusses the design of Pubblicamente, a newly created Italian web call centre.
\end{abstract}

\section{Introduction}

For a company a call centre represents the primary way of interacting with customers. Call centres are all about getting customers the information they need, as quickly as possible. To meet this goal, the knowledge resources of a call centre must be managed in a coordinated and integrated way.

A key ingredient in call centre success is knowledge. The greatest challenge of running a call centre is ensuring that customers are provided with the right information in a timely fashion.

A call centre is confronted with a number of considerable challenges. Staff members answer a wide range of customer questions, drawing on a large body of knowledge. With the high rate of staff turnover, the training required to impart this information can be a considerable burden on call centre resources. Employees are also expected to provide fast answers to customers, even though there is legal accountability for the information they provide. This is further compounded by the high stress work environment and the emphasis on reducing call-handling times. Knowledge management can be used to meet many of these challenges. These include reducing training time and improving the consistency and accuracy of information provided to customers.

The basic tenet is that work in call centres has to be conceptualized in terms of distributed knowledge. This means that only part of the knowledge needed to carry out any transaction is (or rather has to be) in the mind of the operator, and important knowledge may be distributed among colleagues in the organization, available and accessible cognitive artifacts in the work environment, and clients.

The most effective way of tackling the knowledge challenges of a call centre is to develop a knowledge base that contains all the information needed by the call centre staff.

The original version of this chapter was revised: The copyright line was incorrect. This has been corrected. The Erratum to this chapter is available at DOI: 10.1007/978-3-540-44836-5_33

M.A. Wimmer (Ed.): KMGov 2003, LNAI 2645, pp. 192-202, 2003.

(C) Springer-Verlag Berlin Heidelberg 2003 
In a call centre efficient processes must be put in place to ensure that the right knowledge is captured, managed, and kept up-to-date and knowledge management systems must be established to support these processes.

Classical call centres, focused on the effectiveness and efficiency of handling telephones calls, are now transforming towards web call centres. A web call centre is a system that integrates voice, e-mail, and web services. More than traditional call centres, a web call centre provides its users a rich set of interactive services that can be exploited by seamlessly combining the best of both traditional and new media communications.

To achieve these goals a web call centre has to communicate to a large set of users through different channels. For example, in case of need a visitor of a web site can interact with a special operator in many ways including e-mail, web callback, Voice over Internet Protocol (VoIP), and video. Each communication channel has access to a shared information system that thus becomes the most important resource of the entire system.

This work presents the design of an Italian web call centre. Section 2 discusses the nature and the characteristics of a web call centre. The newly created Italian web call centre Pubblicamente is presented in Section 3. Section 4 shows a comparison between Pubblicamente and similar web call centres. The paper ends with future development of Pubblicamente.

\section{Web Call Centre Characteristics}

The realization of a web call centre requires relevant investments in technology, to assure a rich set of services at a high quality level, and in training. A variety of new and emerging information and communication technologies are enabling this change in service delivery.

Some actions have to be carry out to implement a call centre such as:

- Consult with target groups to identify demand for particular services and types of access;

- Plan the type of delivery mechanism. This will help to identify back office functions that may need to be tied together to deliver services in the future;

- Clearly deliver the information system and more importantly, let target groups know of its existence;

- Define a structured and usable knowledge repository that can be used in a number of practical ways: publishing a selection of customer questions (FAQs) to the website, ensuring that on line information is accurate and comprehensive (this will reduce the number of calls in these areas), developing standard responses to a range of common e-mail or queries. Products even exist that will analyse incoming e-mails and automatically select appropriate responses;

- Monitor service delivery, manage its ongoing provision and identify areas in which it can be enhanced either through the addition of other internal services or through the joining up with others.

Adopting a wider range of contact media for dealing with citizens and business in the contact centre, which is ideally a highly structured environment for dealing with contacts, potentially makes it easier for citizens or business to be supplied with a consistent high-quality service [2]. 


\subsection{Quality Control}

To achieve appropriate and predefined levels of service it is necessary to implement a quality system. The main goal of the quality system is to define and control both the quality of the information given by the web call centre and the quality of its services.

The quality of the information given by a web call centre can be expressed in terms of three properties!

- Consistency: similar documents are classified in the same way;

- Completeness: the presence of all relevant documents in each category;

- Accuracy: how properly a document is assigned to a category.

The assessment of information quality allows, in case of evident insufficiency, to activate corrective actions. Thesaurus characteristics, such as uniformity, precision, and dimension, are used to assess efficacy.

The quality of the service of a web call centre is assessed according to consolidated standard and parameters, such as:

- Efficiency: expressed in terms of productivity, response time, services utilization rank; efficient processes must be put in place to ensure that the right knowledge is captured, managed, and kept up-to-date;

- Effectiveness: the number of supplied services, users number, info update, service continuity, answer time;

- Quality: expressed as awaiting reply, processing reply,

- Transparency (with users), customer satisfaction;

- Accessibility: as navigability of web pages, accessibility to disabled people interaction, security, ease of use, reliability, utilities, standards.

\section{The Project Pubblicamente}

Progetto integrato call centre e servizi informativi per l'innovazione della pubblica amministrazione, started in December 2000 with an agreement between Formez and Dipartimento della funzione pubblica, is aimed to realize a web call centre (called Pubblicamente [3]) to deal with all major community inquiries about Local Public Administration (PAL) innovation sector, with a focus on high-volume inquiries such as access to government community support programs, access to government laws, regulations, procedures, PAL "help line" - to help the community identify the right source to particular information needs.

The goal of Pubblicamente, built using phone and Internet technology, is to become a single point of access to manage all enquiries relating to defined areas of information.

The web call centre also aims at establishing a well-defined interface between the expected users and the Formez experts.

1 This definition is given by Information Quality and corresponds to the Centre for Information Quality Management (CIQM) definition. 


\subsection{Characteristics of Pubblicamente}

Three operative sections, a Management Committee and other associations to exiting reality form the system:

- Scouting and operative coordinating group;

- Knowledge management and intermediation system;

- Front-office structure (help-desk and vortal)

- Management Committee to supervise development politics and services supply.

The scouting group researches and mapping exiting information sources, locates of information lack as to thematic areas, implements of knowledge base (KB), monitors of information demand.

To carry out its activity, tSEQhe scouting group is using an information system (KB-SI) with two databases (KB-BD and KB-SD) and a search engine (KB_MR) to search into the web.

In the first phase of Pubblicamente start-up plan the bases of knowledge base have been made. The KB is formed by first level information (data and documents) and second level information (external system). In the second phase the monitoring demand has been started to orient scouting group activities.

\subsection{Functionality}

Pubblicamente is organized in a public area and in a private area where it is possible to access a database of analysed and validated information. Both areas supply the following functionality: access to the glossary, navigate, through the glossary, inside a vertical classification of the thematic areas defined in the database, search, both in simple and advanced mode, driven by the glossary, send questions (only registered users).

In order to access the private area of Pubblicamente a user must be registered. After being registered, the user can submit a question to Pubblicamente by using one of the following channels: web site, telephone, e-mail, fax, mail.

The output channels used by Pubblicamente to reply are the same of the input channels plus the newsletter. Generally the answering time at first level operators is between 5 and 10 minutes. Questions forwarded to a Formez expert require an answering time that ranges between 24 and 48 hours. At the moment, non-registered users can have access only to not validated documents.

The operators that work at the contact centre, use two communications channels:

- The help-desk that manages incoming requests with standard methods, assuring appropriate answering time and monitoring the public interaction,

- The vertical portal (vortal) that allows direct interrogations and access to different communications channels and call centre sources.

The operators use a search engine to reply questions: the search engine does not deliver the complete solution, but provide structured and meaningful browsing and navigation methods. In fact it presents the correct items and the related pages. 


\subsection{System Database}

Pubblicamente provides a flexible and high quality access to the widest range of PAL web resources by a structured database and a quality system.

System database is an on line information resource that is comprehensive, accurate and up to date, well structured, easy and efficient to use, supported by tools for searches and indexing, created and maintained by scouting group.

The database includes information (documents, magazines, web site pages, and public administration norms) of analyzed databases, FAQ and web site as PARete [4]. At the moment 3.523 documents and 424 institutional organizations (universities, departments, Public bodies, Local bodies) have been classified. The database is connected to others Formez databases, but to solve the problem of uniformity of the structures of databases they treat them as external sites.

The precise nature of the information to be provided, and the target audience of the information, has been determined by users needs and research on interest sites. Pubblicamente arise from such an approach would be driven by community information needs, rather than departmental information storage ownership.

To ensure that consistent responses are provided to operators, a repository of second level support teams and contact centre common problems and resolution have been developed. This information is an excellent source and will reduce the number of simple calls to contact centre. At the moment these repository is not on line, but only used by operators.

Develop Knowledge Management System. To collect information for the database the researchers have defined a taxonomic scheme [5], based on a top-down approach.

Taxonomic Scheme Definition. The taxonomic scheme is useful to structured search in areas and sub-areas or driven by glossary key words.

To develop the taxonomic scheme some activities have been carried out such as analysis of Formez web site and its thematic sections, analysis of others Central and Local Public Administration sites, analysis of others taxonomic scheme, individuation of interest areas and macro-areas, integration of all analyzed schemes in a unique taxonomic scheme, definition of relations between terms.EINBETTEN

At the moment there are 14 different thematic areas. The thematic areas could be modified: if there are not references in a thematic area, the scouting group begins to analyse this cause. There are two causes:

- Users information needs do not concern that area. Perhaps it is necessary include that area in an another one or erase it;

- The name of area is wrong and so only few documents can be catalogued.

This operation is periodically made by scouting group to provide a validated and revised research method, based on thematic areas.

The glossary. The taxonomic scheme has been analyzed to define glossary terms. Usually there are difficulties to successfully retrieve information on advanced alternative methods in on line databases. For this reason, a thesaurus is used to locate information in data retrieval systems. 
The thesaurus is a controlled particular sector terms vocabulary arranged in a know order, with specified types of semantics relationships. The structure of the thesaurus is [6]:

- Keyword: terms used to search the site by queries;

- Thematic area: the areas associable to keywords used for directory hand browsing;

- Definition: sharpening the sense of the term;

- UF (used for): keywords synonyms;

- BT (broader term): the super ordinate term, i.e. the one that identifies the following terms as broader terms to the heading term;

- NT (narrower term): the subordinate term, i.e. the one that identifies the following terms as narrower terms to the heading term;

- RT (related term): the terms in a logical relation to another keyword.

Keywords enable more precise retrievals. Thesauruses find their main application in large literature databases.

As the goal of Pubblicamente is to reach Public Administration, the scouting group has developed a specialised thesaurus ensuring the use of appropriate and homogeneous terms, classification schemes and topics covered. So the thesaurus is the basis for indexing and retrieving material.

To ensure appropriateness of the chosen terms, a methodology has been used. Some methods have been based on actual phrases, which occur in documents and should therefore reflect the preferred terminology of the authors of the articles. Pubblicamente thesaurus is, therefore, built out of the text and not added on. It will consist of key terms, which could usefully be used to search within any database [7].

List of words and phrases will be extracted by computerised analysis of original documents.

Schedule. Pubblicamente thesaurus has been developed in the following step:

1. First definition (July of 2001): development of the glossary by a bottom-up approach directed at sites, literature and documents about Public Administration. Each defined thematic area has been sound to find reference terms. The first version of the glossary has been confronted with key words found in the PA sites. Then a keyword glossary has been made and the keywords inserted in the thesaurus, with synonymous, related, broader and narrow terms. This first version has been circulated to a selected number of experts in the various fields for comments and suggestions.

2. Evaluation of the method (February 2002): the method has been evaluated considering if the used approach could create a complete thesaurus in all fields of Public Administration area;

3. Thesaurus updating and maintenance (after April 2002): based on the outcome of item 2, a second circulation is foreseen to a broader selection of experts with particular emphasis on new topics. A priority setting will be necessary before circulation. Periodically researchers insert new terms of glossary, basis on user demands and others research on interest sites and documents, and associate documents to them.

The population of glossary has to be made incrementally, inserting the complex terms definitions and then the missing definitions. The research could be made using research engines, on line vocabulary and norms definitions. 
During the development of glossary, the related, narrow, and broader terms are consider keywords, thematic areas are not keywords and keywords must be inserted in the sub-areas (of main thematic areas).

In order to define terms form and meaning, the following editorial and conventional rules have been established:

- Abbreviations are synonyms, without punctuation and in lower-case;

- Composed names have only the first letter in upper-case;

- Foreign terms are not inserted.

An example. All the entries in the Pubblicamente database are catalogued by keywords listed in the thesaurus. Once selected a keyword, user can look for keywords that are related or more specific. Once he has found the relevant keywords, type them in the search engine form below the thesaurus.

Each subject field is expressed by a very broad concept, a top term. From these top terms chains of progressively more detailed descriptors develop downwards to most specific issues. Some issues at the same level of a particular structure may be related, but not dependent upon each other

If you click on a term in the combo box with thematic areas, the results page shows the thematic sub-areas and the term of glossary related to that area. To find information, you have to click on a term and a news page with documents and related sites, broader, narrow and related terms appears.

\subsection{Information Quality}

The analysis has been carried out on taxonomic schema. In order to define the quality of the taxonomy we need metrics to estimate the correctness of the hierarchical structure, the consistency of the terms, the degree of the hierarchy, the suitability of terms RT, UF, NT and BT. So we used the following metrics:

- Dimension, i.e., the number of areas, sub-areas, terms, organizations and resources;

- Terms distribution.

The metrics have been computed by some SQL queries (written in Microsoft Access). We use these metrics to monitor the growing rate of the collected material and the balancing of the taxonomic scheme:

- NUMKWperArea, returns the number of KW of each area;

- NUMBTperKW, returns the number of BT of each KW;

- NUMRTperKW, returns the number of RT of each KW;

- NUMNTperKW, returns the number of NT for every KW;

- NUMUFperKW, returns the number of UF of each KW;

- NUMBT, returns the events of every BT in the thesaurus;

- CorrispAreeNonSame, returns the number of KW, NT, BT, UF of each without duplicates;

- PopolamentoGauss, returns the number of areas with values in fixed ranges.

In order to analyze the dimension of the taxonomy and study its distribution we use a histogram having on the $\mathrm{X}$-axis the data and on the $\mathrm{Y}$-axis the measured values. The steps to build a distribution histogram are the following:

1. Define the population intervals to calculate the distribution;

2. Write on the $\mathrm{X}$ axis the term intervals; 
3. Write on the Y-axis the number of areas, of unitary dimension, for the intervals defined on the $\mathrm{X}$-axis.

These histograms let us represent the number of terms BT, NT, UF, RT identified for each term KW, and obtain the indicators of generalization, specialization, synonymy and aggregation of the taxonomic scheme. Figure 1 shows the result of NUMKWperArea formula and NUMKWperArea histogram.

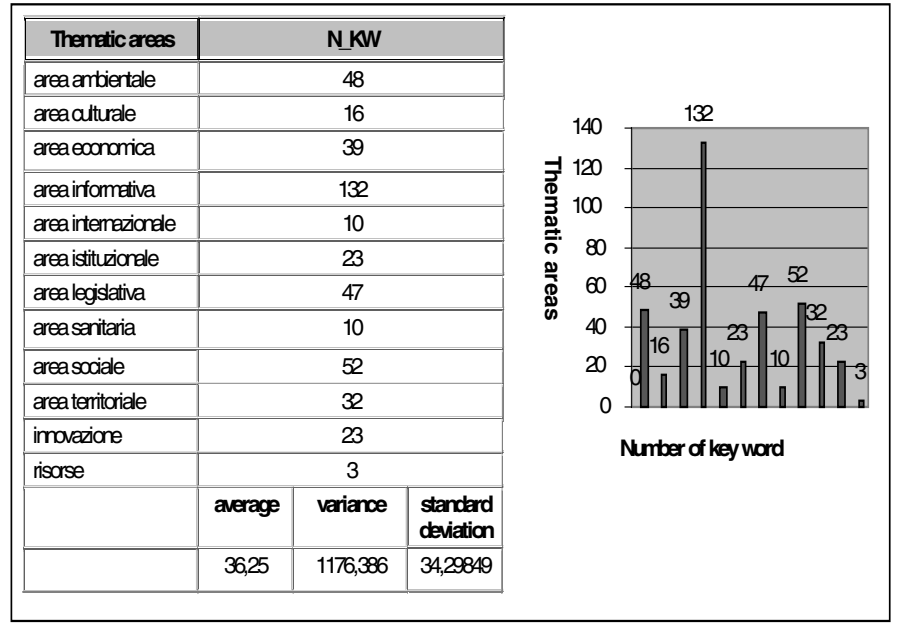

Fig. 1. NUMKWperArea, NUMKWperArea histogram

Further results for quantitative analysis of the taxonomic scheme are obtained:

- Applying the formulas for the average calculation, the variance and the standard deviation with respect to the query results;

- Comparing the histogram results with the average of the corresponding values;

- Analyzing the dispersion of the values.

In the initial phase it is recommended to have a uniform distribution of the terms in the thematic areas to be able to answer simple questions. Afterwards, as new requirements appears, it is possible to refine and expand specific areas.

\subsection{Service Quality}

To attain appropriate call centre levels of service and to assure usability and accessibility sites requirements, a system quality has been defined.

After identifying quality indicators and twenty-three levels of services, any indicators have been associated to corresponding level of service to control each considered aspect. Then each level of service has been specified, dynamically, according to the process phase. There are two phase:

- Start-up phase, i.e. initial supplying phase in which data are periodically analysing to define levels of threshold;

- Working phase, when used levels of service are defined according to call centre needs and are continually controlled. 
Variables represent the levels of threshold. According to the phases, they will assume different values.

To define the right corrective actions if the service level is not observed is necessary define causes, detailing call centre interactions steps, associate statistical components to interactions steps, compute medium time of each step, define limit cases, compute the value of the levels of threshold according to each step medium time and limit cases.

Ishikawa's diagram [8] is used to analyze the causes when the service level is not observed. The level of threshold is on the horizontal row and the causes are on the transversal line. Then the causes are grouped in classes and ordinate, approach to major o minor probability to show up. So the right corrective actions have been defined.

Figure 2 presents an example of computed Ishikawa's diagram.

An example. We can analyze the level of service: Daily Calling Percentage at first level by phone. This value has to be less or equal to the following level of threshold:

$$
\text { Start-up phase: } S 1_{s}=C 1 \% \text { of the total of calling }
$$

$$
\text { Operating phase: } S 1_{f}=C 2 \% \text { of the total of calling, with } C 1>C 2
$$

We assume a higher value in the start-up phase because the users will use phone instead of the other channels. In the working phase, this value will decrease following the increase of the Internet requests. To decrease this value is necessary a good operator training, an important service dissemination phase and the observation of the other access channels levels of service. The following Ishikawa diagram analysis these factors, showing the violated level of threshold and the causes.

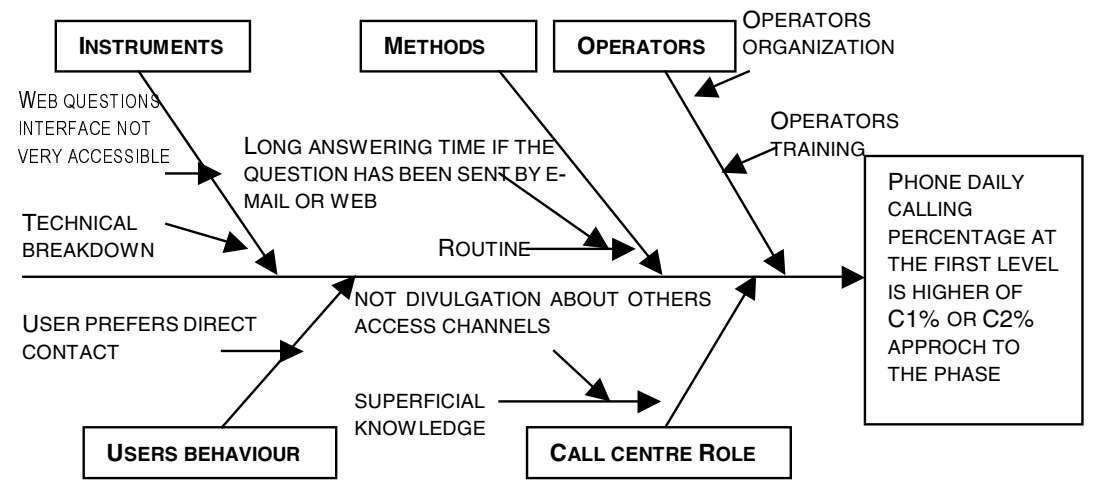

Fig. 2. An Ishikawa diagram

\section{Comparisons to Other Similar Experiences}

In several countries call centres are an important interface in the management of government relationships with citizen and business. In fact, citizens greatly value timely accessibility to government information that affects their lives. In fact, the vision of the citizen e-government center of the future is to allow citizens seamless 
access to government information at any time, from anywhere, in any form, and for free. Often a call centre is the first point of contact with government.

Some researches highlight that, in the next few years, government at each levels will be moving their low-tech call centres from back-office support to the front-line of the agency. In this migration, the call centres are being outfitted with the latest in high-tech hardware and software in both voice and data applications [9]. The focus is moving from only telephone calls to all forms of citizen access, including e-mail, faxmail, kiosk, and the Internet.

We have analyzed some web call centre in the world to find similar characteristics to Pubblicamente, but Service Tasmania, in Australia, has the same key objectives, in spite of it presents a more articulated structure.

Service Tasmania [10] provides a flexible access to the widest range of web resources, primarily information and services from the State Government, but also from the Federal and local governments. The web site groups information and transaction services according to user needs, so that Tasmanians no longer need to know how government is structured to find what they want. Finally, if they do not find the required information on the site, they can contact a web call centre and receive back an answer by mail, phone, fax, or e-mail.

In order to improve the quality of the on line services, user behavior has been monitored and analyzed during three months, even using an help desk system operating via e-mail. The most frequently research keywords and the popular sections of the web site have also been identified. Also user preferences are analyzed and a special attention is devoted to unsuccessful queries.

The characteristics of Service Tasmania user-centric model are: the user sees government information as a simple and homogeneous collection of information that features a variety of browsing paths and discovery strategies (behavior), suitable for different kind of needs and capable to adapt to different user skill and education.

Pubblicamente and Service Tasmania supply a flexible access to the multitude of government information and develop a database to allow simple data insertion and maintenance operations. Although they operate in an environment where content continues to grow rapidly and where there is little or no control over development and quality content, major strategies have developed to enable the user to locate or discover specific content.

Both call centres use tools for automatic validation of the presence of sites after routine harvesting, and the exclusion of missing sites and annex of national and international standards, whenever possible.

Pubblicamente and Service Tasmania sites allow users to search information and services in a variety of ways.

Service Tasmania provides a navigation structure which allows user to search information and services with cross-linkages at various points to other views of the data, and with contextual free text searching available throughout.

The registered user of Pubblicamente can search information on the web site consulting the glossary, navigating with the glossary, in a thematic area or with simple and advanced research of information with insertion of glossary terms.

The development of these call centres is based upon a number of additional requirements of a technical nature including the capacity to cope with large amounts of data and a high site demand.

Quality assurance and standard adherence are fundamental to the operations of any call centre examined. 


\section{Conclusions}

Call centres are all about getting customers the information they need as quickly as possible. To meet this goal, the knowledge resources of a call centre must be managed in a coordinated and integrated way.

In this paper we highlight the potential of call centres to meet PAL interest areas, but also the policy and management challenges they present.

This paper presents a framework that defines the essential characteristics of a web call centre such as:

- Use Web-based technologies wherever possible because these can be integrated into a single and consistent interface for users, which give greater efficiency;

- Give operator searching and browsing methods and support these with comprehensive meta-data, such as titles, descriptions and keywords;

- Search the raw information and shape this into a structured and usable knowledge repository;

- Use statistics to measure and track the success of the knowledge base.

In this work we have provided at least an example of a project to develop a PAL call centre that is suitable for other target call centre.

A further improvement of the project is expected from the introduction of XML to describe documents with meta-data and the development of a search engine on XML information.

\section{References}

1. Sebastiano Bagnara, "Euro-telework: Report on call centres", http://www.telework-irti.org/bagnara.htm

2. "Call Centres in the Scottish Public Sector" http://www.scotland.gov.uk/government/c21g/Call_centre_c21g.pdf

3. Pubblicamente web site, http://www.pubblicamente.it

4. PARete, http://www.di.unipi.it/parete

5. Marcello Giacomantonio, "Image Cataloguing and Didactic Management of a iconic file" (In Italian), Quaderno di comunicazione audiovisiva (in Italian)

6. Thesaurus on Advanced Alternative Methods (TAAM), http://ecvam-sis.jrc.it/cover/thesaurus.html

7. Marcello Giacomantonio, "Computerizing of Recording System" (in Italian), Quaderno di comunicazione audiovisiva, 8: 10-21, anno 3.

8. Ishikawa's diagram, http://mot.vuse.vanderbilt.edu/mt322/Ishikawa.htm

9. Purdue Research Foundation, "Government Call Centers Performance Benchmark Report, Final Results", http://www.amsinc.com/crm/govtcallcenterstudy.asp

10. Service Tasmania web site, http://www.servicetasmania.tas.gov.au 